\title{
MODELLING OF ACCOUNTING INFORMATION SYSTEM FOR MILITARY MEDICAL INSTITUTIONS
}

\author{
Ivan M. Milojević \\ University of Defence in Belgrade, Republic of Serbia \\ Rosa M. Andžić \\ Alfa BK University, Belgrade \\ Svetlana D. Ignjatijević \\ University Business Academy in Novi Sad
}

\begin{abstract}
The complexity of the accounting system as the subsystem of management information system requires the continuous arrival of different types of data in different intervals which leads to an increase in complexity during its processing and presentation to management. Promoting the work and operations of military medical institutions requires the use of just such modern accounting information systems. Methods - Queuing system is used in this paper as the basis for positioning of information which needs to be processed within the accounting process, by adhering to certain limitations which are imposed by the specificity of the military medical system which is established. Conclusion - Basis of this information system is comprised of three subsystems, which are financial accounting, managerial accounting and cost accounting. Conditioning processing of data in the accounting process, adhering to accounting principles, primarily the principle of realization, it is very significant that this system supports information which is objectified in the form of accounting documents, so it can be processed and given to its users u military medical institutions.
\end{abstract}

Key words: modelling, accounting, information system, queuing system

\section{Introduction}

$\mathbf{Q}$ usiness records are kept in order to cover all of the needs of individual subsystems of companies i.e. for their guidance and rational spending in accounting information system for military medical institutions. In contemporary conditions, despite the obviously significant results which are achieved by accounting information systems in for military medical institutions, efficiency problems are more pronounced. They are manifested in inhomogeneity and disintegration of the system, mostly from the perspective of untimely information, based on which decisions from management are made. 
By establishing a unique accounting information system within a military medical institution, a sound basis for bringing timely information for managers is created. It is important to establish quantitative in addition to the qualitative dimension of this system, which is reflected in the ability to bring in focus the accounting process i.e. the processing system which is called bookkeeping, depending on the size of the set of accounting data which need to be processed.

It is very important, by cognition of elements of this system by applying quantitative methods, for the queuing system in this case to show the possibility to process certain amount of data within the accounting flow which would result in timely and high quality information which would be used in the decision-making process.

\section{Description of the Queuing System}

Today the queuing systems have wide applications[1], and are used to automate the process of operating calculation [2] and distribution of limited (and highly variable in time) resources among many consumers[3]. The system performs servicing of stochastic flow requirements, which includes data on the type and amount of required resources, $\beta$ needed resource, as well as determining the ability to meet every requirement of the system in military medical institutions [4].

One of the basic tasks that arises in the design of such queuing systems, is finding optimal management [5] by allocation of resources on stochastic [6] flow requirements. Finding the optimal discipline of queuing [7] is of great importance, as it maximizes revenue from servicing requests of military medical institutions [8].

\section{Setting of the Problem and Marketing}

Let us assume that in the servicing accounting system[9], which is comprised of $m$ amount of business records, that are $\alpha_{1}, \alpha_{2}, \ldots, \alpha_{m}$ units respectively, arrives an $n$ amount of independent stationary input-output accounting data[10] of Poisson's flow of demands, with parameters $\lambda_{1}, \lambda_{2}, \ldots, \lambda_{n}$.

The complexity of the accounting system requires the arrival of different types of information [11] at different time intervals, which complicates the problem.

If $\beta_{i j}$ - is the number of units of $j$ type, which requires the request of $i$ type $(i=\overline{1, n}$; $j=\overline{1, m}$ ); in that way, the request of $i$ type requires the resources which, in quantitative terms[12], are written in the following way: $\beta_{i}=\beta_{i_{1}}, \beta_{i_{2}}, \beta_{i_{m}}$

Such needs are, either met or not met in full (partially met demands are excluded) [13]. In case of servicing the requests $i$ type, the system receives revenue $C_{i}$. The task consists in finding the optimal management of resource allocation[14], which maximizes the expected usefulness of accounting information[15] from servicing requests during a given time interval $T$. 


\section{Model of the Solution to the Problem}

Static algorithm [16] of resource allocation assumes processing of accounting data in chronological order of requests with the principle of "first came - first served". (Figure 1)

In solving the task, we will limit to the case when $\alpha_{1}=\alpha_{2}=N$ and the matrix

$$
\left\|\beta_{i j}\right\|=\left[\begin{array}{lll}
1 & 1 & 0 \\
0 & 1 & 1
\end{array}\right]
$$

which has significant practical applications.

We will show the assumption of the solution of three parts of the accounting information system for military medical institutions, financial, managerial and cost accounting [17].

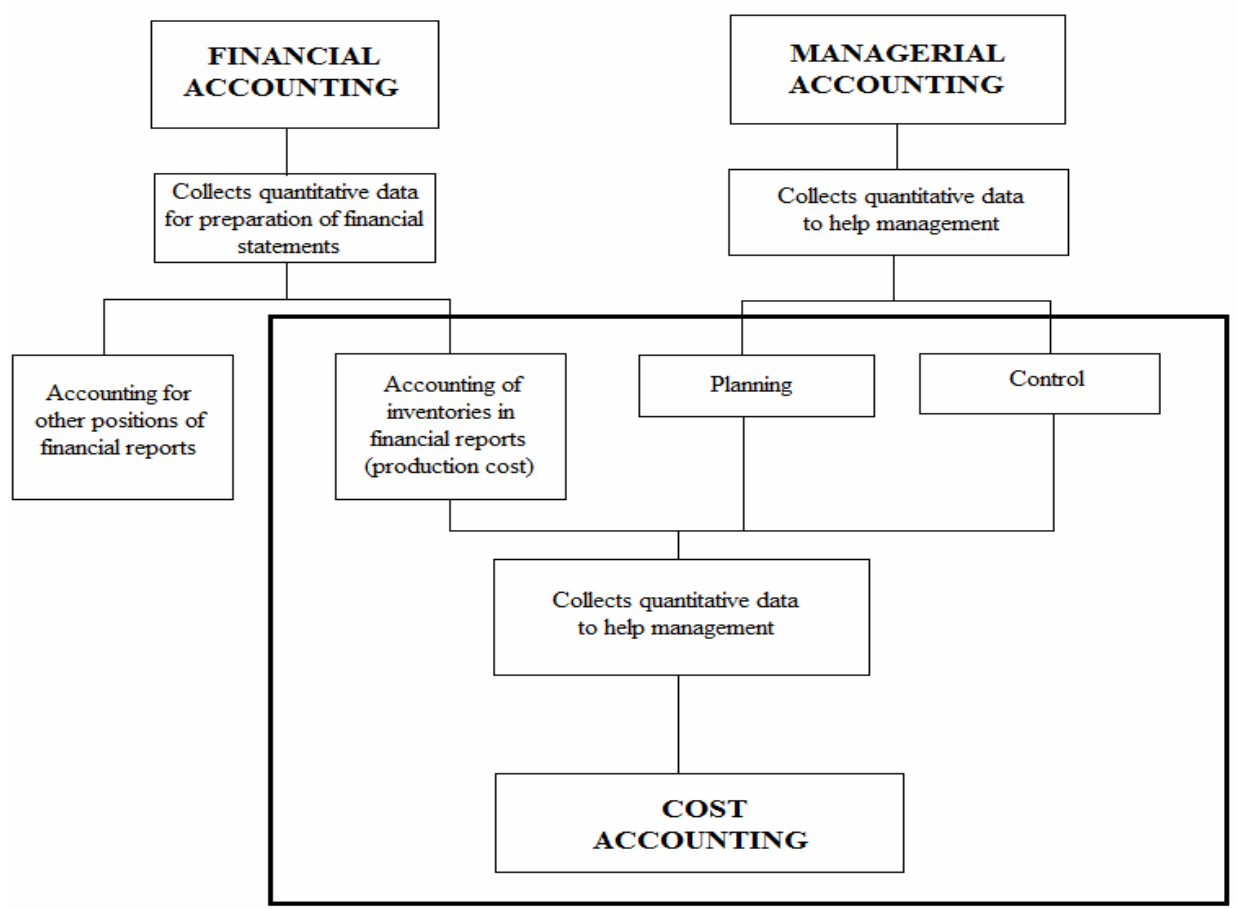

Figure 1 - Accounting system

If $P_{i j k}(t)$ is the probability that, in the moment $\mathrm{t}$, exactly $i$ requests of the first flow $i=\overline{1, N}, j$ requests of the second flow $j=\overline{1, N}$ and $k$ requests of the third flow $k=\overline{1, N}$ are satisfied. Let's show the state of the system in the form of the following 
sets: $\quad i+j<N, k+j<N ; \quad i+j<N, k+j=N ; \quad i+j=N, k+j<N$; $i+j=N, k+j=N$;

When $i+j<N, k+j<N$; , due to independence of handling requests of different flows, we have

$$
P_{i j k}(t)=\frac{\lambda_{1}^{i} \lambda_{2}^{j} \lambda_{3}^{k}}{i ! j ! k !} t^{(i+j+k)} e^{-\lambda_{t}}
$$

Analogously when, $i+j<N, k+j=N$; we get

$$
P_{i j k}(t)=\frac{\lambda_{1}^{i}}{i !} e^{-\lambda_{1}^{t}} P_{j k}(t)
$$

where $P_{j k}(t)$ is the probability that, in the moment $t$, exactly $j$ requests of the second flow are satisfied, and $k$ requests of the third flow (provided that $k+j=N$ ). The probabilities of $P_{j k}(t)$ satisfy the following system of differential equations:

$$
\frac{d P_{j k}(t)}{d t}=\lambda_{2} P_{j-1, k}(t)+\lambda_{3} P_{j, k-1}(t)
$$

Denote by

$$
\hat{P}(s)=s \int_{0}^{\infty} P(t) e^{-s t} d t
$$

Then

$$
\hat{P}_{j k}(s)=\frac{\lambda_{2}^{j} \lambda_{3}^{k}(j+k-1)}{(j-1) ! k !} \frac{1}{\left(s+\lambda_{2}+\lambda_{3}\right)^{j+k}}+\frac{\lambda_{2}^{j} \lambda_{3}^{k}(j+k-1)}{j !(k-1) !} \frac{1}{\left(s+\lambda_{2}+\lambda_{3}\right)^{j+k}}
$$

Further, by replacing the probability (2) we obtain

$$
\begin{aligned}
& P_{i j k}(t)=\frac{N !}{i ! j ! k !} \frac{\lambda_{1}^{i} \lambda_{2}^{j} \lambda_{3}^{k} t^{i}}{\left(\lambda_{2}+\lambda_{3}\right)^{N}} \\
& i+j<N, k+j=N ; \\
& {\left[e^{-\lambda_{1 t}}-e^{-\lambda t} \sum_{r=0}^{n-1} \frac{\left[\left(\lambda_{2}+\lambda_{3}\right) t\right]^{r}}{r !}\right]}
\end{aligned}
$$


Analogously we find:

$$
\begin{aligned}
& P_{i j k}(t)=\frac{N !}{i ! j ! k !} \frac{\lambda_{1}^{i} \lambda_{2}^{j} \lambda_{3}^{k} t^{k}}{\left(\lambda_{1}+\lambda_{2}\right)^{N}} \\
& i+j=N, k+j=N ;\left[e^{-\lambda_{3 t}}-e^{-\lambda t} \sum_{r=0}^{n-1} \frac{\left[\left(\lambda_{1}+\lambda_{2}\right) t\right]^{r}}{r !}\right]
\end{aligned}
$$

It remains to determine the probability $P_{i j k}(t)$, where $i+j=N, k+j=N$; we have

$$
\frac{d P_{i j k}(t)}{d t}=\lambda_{1} P_{i-1, j, k}(t)+\lambda_{2} P_{i, j-1, k}(t)+\lambda_{3} P_{i, j-1}(t)
$$

where $P_{i-1, j, k}(t), P_{i, j-1, k}(t), P_{i, j .-1}(t)$ are determined, respectively from the formulas (4), (5), (1).

Solving the system of equations (6), we get:

$$
\begin{aligned}
& P_{i j k}(t)=\frac{\lambda_{1}^{i} \lambda_{2}^{j} \lambda_{3}^{k}}{j !}\left\{\frac{N !}{\left(\lambda_{2}+\lambda_{3}\right)^{N} k ! \lambda_{1}^{i}} \rho\left[i-1, x_{1}\right]+\frac{N !}{i !\left(\lambda_{1}+\lambda_{2}\right)^{N} \lambda_{3}^{k}} \rho\left[k-1, x_{3}\right]+\frac{j(N+K-1) !}{i ! k ! \Lambda^{N+k}}\right. \\
& \rho[N+k-1, x]-\frac{N !}{i ! k !} \sum_{r=0}^{N-1}\left(\frac{i\left(\lambda_{2}+\lambda_{3}\right)^{r-N}(r+i-1)}{r ! \Lambda^{i+r}} \rho[r+i-1, x]\right. \\
& \left.\left.+\frac{k\left(\lambda_{1}+\lambda_{2}\right)^{r-N}(k+r-1) !}{r ! \Lambda^{k+r}} \rho[k+r-1, x]\right)\right\}
\end{aligned}
$$

where

$$
\rho[y, z]=1-\sum_{m=0}^{y} \frac{z^{m}}{m !} e^{-z} ; x_{i}=\lambda_{i} t(i=1,2) ; x=\lambda t
$$

The expected usefulness of the information obtained from the accounting information system for military medical institutions measured by the degree of significance for managerial decisions[18] $V_{N, N}(t)$, which will be got by the system while servicing, by the principle "first came - first served" [19], has the form:

$$
V_{N, N}(t)=\sum_{u, j, \kappa} P_{i, j, k}(t)\left[c_{1} i+c_{2} j+c_{3} k\right]
$$


In order to investigate the stationary regime, let us consider the second form $V_{N, N}(t)$. According to the formula of expected value, we have

$$
V_{\alpha}(t)=\sum_{r=1}^{3} \int_{0}^{t} \lambda_{r} e^{-\lambda u}\left[c_{r}+V_{\alpha}-\beta_{r}(t-u)\right] d u
$$

where

$$
\alpha=\left\{\alpha_{1}, \alpha_{2}\right\}, \lambda=\sum_{t=1}^{3} \lambda_{t}
$$

By differentiating (7) we get:

$$
\frac{d V_{\alpha_{1}, \alpha_{2}(t)}}{d t}=\sum_{t=1}^{3} \lambda_{i} c_{i}+\lambda_{1} V_{\alpha_{1}, \alpha_{2}(t)}+\lambda_{2} V_{\alpha_{1}-1, \alpha_{2}-1(t)}+\lambda_{3} V_{\alpha_{1}, \alpha_{2}-1(t)}=\lambda V_{\alpha_{1}, \alpha_{2}(t)}
$$

In the stationary regime, when $t \rightarrow \infty$, we have

$$
V_{\alpha_{1}, \alpha_{2}}=R+\alpha V_{\alpha_{1}-1, \alpha_{2}}+\beta V_{\alpha_{1}-1, \alpha_{2}-1}+\gamma V_{\alpha_{1}, \alpha_{2}-1}
$$

the degree of significance gives priority in processing of the data over their entrance into business records, traditional $\leftrightarrow$ modern approach, where:

$$
R=\frac{1}{\lambda} \sum_{i=1}^{3} \lambda_{i} c_{i} ; \alpha=\frac{\lambda_{1}}{\lambda} ; \beta=\frac{\lambda_{2}}{\lambda} ; \gamma=\frac{\lambda_{3}}{\lambda}
$$

Let us introduce the production function:

$$
\rho_{\alpha_{2}}(z)=\sum_{\alpha_{1}=1}^{\infty} V_{\alpha_{1}, \alpha_{2} z} \alpha_{1}
$$

Multiplying both sides of the equation (8) with $z^{\alpha_{1}} i$ and summing by $\alpha_{1}$, from 1 to $\infty$, we get

$$
\rho_{\alpha_{2}}(z)=R \frac{z}{1-z}+\alpha z\left[V_{0, \alpha_{2}}+\rho_{\alpha_{2}}{ }^{(z)}\right]+\beta_{z}\left[V_{0, \alpha_{2}-1}+\rho_{\alpha_{2}-1}\right]+\gamma \rho_{\alpha_{2}-1}(z)
$$

Getting that $V_{0, \alpha_{2}}=c_{3} \alpha_{2}$, we have the recurrent relationship

$$
\rho_{\alpha_{2}}(z)=\frac{z}{(1-z)\left(1-\alpha_{z}\right)}-c_{3} \beta \frac{z}{1-\alpha_{z}}+\alpha_{2} c_{3}(\alpha+\beta) \frac{z}{1-\alpha_{z}}+\rho_{\alpha_{2}-1}{ }^{(z)} \frac{\beta_{z}+\gamma}{1-\alpha_{z}}
$$


where:

$$
\rho_{0}(z)=\sum_{\alpha_{1}=1} V_{\alpha_{1}, 0} z^{\alpha_{1}}=\sum_{\alpha_{1}=1} c_{1} \alpha_{1} z^{\alpha_{1}}=c_{1} \alpha_{1} \frac{z}{(1-z)^{2}}
$$

Let us denote:

$$
A(z)=\frac{R_{z}}{(1-z)\left(1-\alpha_{z}\right)}-\frac{c_{3} \beta_{z}}{1-\alpha_{z}} ; B(z)=c_{3}(\alpha+\beta) \frac{z}{1-\alpha_{z}} ; a(z)=\frac{\beta_{z}+\gamma}{1-\alpha_{z}}
$$

Then, from the recurrent connection (9) we obtain:

$$
\begin{aligned}
& \rho_{\alpha_{2}}(z)=A(z) \frac{1-a^{\alpha_{2}}(z)}{1-a(z)}+B(z) \frac{\alpha_{2}}{1-a(z)}-\frac{a(z)\left(1-a^{\alpha_{2}}(z)\right)}{(1-a(z))^{2}}+a^{\alpha_{2}}(z) \rho_{0}(z)= \\
& =\left[\frac{A(z)}{1-a(z)}-\frac{B(z) a(z)}{(1-a(z))^{2}}\right]+a^{\alpha_{2}}(z)\left[\rho_{0}(z)+\frac{B(z) a(z)}{(1-a(z))^{2}}-\frac{A(z)}{1-a(z)}\right]+\alpha_{2} \frac{B(z)}{1-a(z)}
\end{aligned}
$$

Taking that

$$
\begin{aligned}
& \frac{A(z)}{1-a(z)}=\frac{R}{\alpha+\beta} \frac{z}{(1-z)^{2}}-\frac{c_{3} \beta}{\alpha+\beta} \frac{z}{1-z} ; \\
& \frac{B(z) a(z)}{(1-a(z))^{2}}=\frac{c_{3}}{\alpha+\beta} \frac{z\left(\beta_{z}+\gamma\right)}{(\alpha+\beta)(1-z)^{2}}
\end{aligned}
$$

we will transform the expression (10) in the form of:

$$
\rho_{\alpha_{2}}(z)=\frac{R-C_{3}(\beta+\gamma)}{\alpha+\beta} \frac{z}{(1-z)^{2}}+a^{\alpha_{2}}(z) \frac{z}{(1-z)^{2}} \frac{\left(C_{1}+C_{3}-C_{2}\right)}{\alpha+\beta}+C_{3} \alpha_{2} \frac{z}{1-z}
$$

Decomposing $\rho_{\alpha_{2}}(z)$ in the series, by degrees $z$, we will get

$$
\begin{aligned}
& \rho_{\alpha_{2}}(z)=\sum_{\alpha_{1}=0}^{\infty} z^{\alpha_{1}}\left[\alpha_{1} \frac{R-C_{3}(\beta+\gamma)}{\alpha+\beta}+C_{3} \alpha_{2}+\frac{\beta\left(C_{1}+C_{3}-C_{2}\right)}{\alpha+\beta}\right] x \sum_{k=0}^{\alpha_{1}} C_{\alpha_{2}}^{k} \gamma_{2}^{\alpha_{2}+k-\alpha_{1}}+\beta^{\alpha_{1}-k} \\
& \sum_{j=0}^{k} a^{j}(k-j) \lambda\left(\alpha_{2}, j\right)+(1-z) \sum_{k=0}^{\alpha_{2}} C_{\alpha_{2}}^{k} \beta^{k} \gamma^{\alpha_{2}-k} \sum_{j=0}^{\alpha_{1}-k} \alpha^{j}\left(\alpha_{1}-k-j\right) \lambda\left(\alpha_{2}, j\right)
\end{aligned}
$$


Where:

$$
\begin{aligned}
& x=\left\{\begin{array}{l}
1, \text { if } \alpha_{1} \leq \alpha_{2} \\
0, \text { if } \alpha_{1}>\alpha_{2}
\end{array}\right. \\
& A\left(\alpha_{2}, j\right)=\frac{\left(\alpha_{2}+j-1\right) !}{\left(\alpha_{2}-1\right) ! j !}
\end{aligned}
$$

From expression (11), with title $\alpha_{1}=\alpha_{2}=N$, follows:

$$
V_{N, N}=N\left(\frac{R-C_{3}(\beta+\gamma)}{\alpha+\beta}+C_{3}\right)+\frac{\beta\left(C_{1}+C_{3}-C_{2}\right)}{\alpha+\beta} \sum_{k=o}^{N} C_{\alpha}^{k} \gamma^{k} \beta^{N-k} \sum_{k=0}^{k} \alpha^{j}(k-j) \Lambda(N, j)
$$

Using (12) we find evaluation results obtained by using the dynamic servicing algorithm, compared with the algorithm of servicing on the principle "first came - first served". (Figure 2)

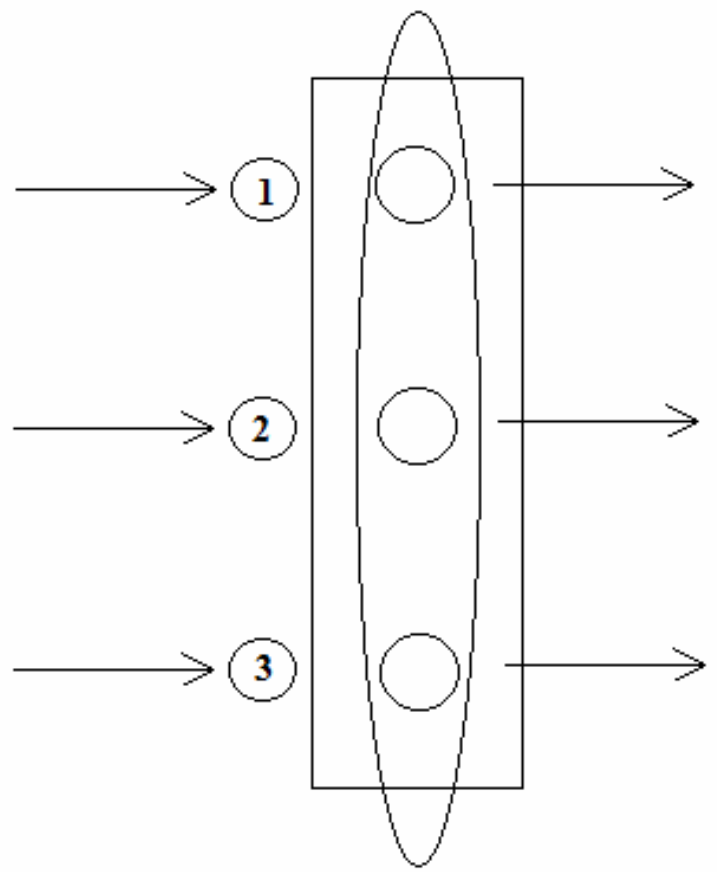

Figure 2 - Accounting process 
The value that the information has for the company gives it the priority obtained by using the dynamic resource allocation algorithm, has the form:

$$
\lambda_{\alpha}(t)=\sum_{r=1}^{3} \int_{0}^{t} \lambda_{r} e^{-\Lambda u} \max \left\{C_{r}+\lambda_{\alpha-\beta_{r}}(t-u) ; \lambda_{\alpha}(t-u)\right\} d u
$$

Let us show that $\delta(t)=\lambda_{N, N}(t)-V_{N, N}(t)$ is a non-declining function. Truly, by differentiating (7) and (13), we get:

$$
\begin{aligned}
& \lambda_{\alpha}^{\prime}(t)=\sum_{r=1}^{3} \lambda_{r} \max \left\{\lambda_{\alpha-\beta_{r}}(t)+C_{\beta_{r}}-\lambda_{\alpha}(t) ; 0\right\} ; \\
& V_{\alpha}^{\prime}(t)=\sum_{r=1}^{3} \lambda_{r}\left\{V_{\alpha-\beta_{r}}(t)+C_{\beta_{r}}-V_{\alpha}(t)\right\} ;
\end{aligned}
$$

From the last two expressions, directly follows that

$$
\frac{d}{d t}\left[\lambda_{\alpha}(t)-V_{\alpha}(t)\right] \geq 0
$$

where by the equality sign is for situations when $t \rightarrow 0$, due to continuity of $\lambda_{\alpha}(t)$ and $V_{\alpha}(t)$ and starting conditions $\lambda_{\alpha}(0)=0, V_{\alpha}(0)=0$. Thus, we have that:

$$
\delta(t)=\lambda_{N, N}(t)-V_{N, N}(t) \leq \lim _{t \rightarrow \infty} \lambda_{N, N}(t)-\lim _{t \rightarrow \infty} V_{N, N}(t)
$$

Supposing (for the purpose of defining) that $C_{1}+C_{3}>C_{2}$, we get the inequality:

$$
\lambda_{N, N}=\lim _{t \rightarrow \infty} \lambda_{N, N}(t)<N\left(C_{1}+C_{3}\right)
$$

Based on the formula (12), we get:

$$
\begin{aligned}
& V_{N, N}=\lim _{t \rightarrow \infty} V_{N, N}(t)>N\left(\frac{R-C_{3}(\beta+\gamma)}{\alpha+\beta}+C_{3}\right)+\frac{\beta\left(C_{1}+C_{3}-C_{2}\right)}{\alpha+\beta} \sum_{k=0}^{N} C_{N}^{k} \gamma^{k} \beta^{N-k} \\
& \sum_{j=0}^{\infty} \alpha^{j}(k-j) \Lambda(n, j)=N\left[C_{3}+\frac{R-C_{3}(\beta+\gamma)}{\alpha+\beta}+\frac{\beta(\gamma-\alpha)\left(C_{1}+C_{3}-C_{2}\right)}{(\alpha+\beta)(1-\alpha)}\right]= \\
& =N\left[C_{2} \frac{\beta}{1-\alpha}+\left(C_{1}+C_{3}\right) \frac{\gamma}{1-\alpha}\right]
\end{aligned}
$$


The given assessment for $V_{N, N}$ is adequate when $\gamma>\alpha$. In the case when $\gamma<\alpha$, assessment has the form:

$$
V_{N, N}>N\left[C_{2} \frac{\beta}{1-\gamma}+\left(C_{1}+C_{3}\right) \frac{\alpha}{1-\gamma}\right]
$$

By replacing (15) - (17) into (14), we get the assessment

$$
\delta(t)<\frac{N\left(C_{1}+C_{3}-C_{2}\right) \beta}{\beta+\max \{\gamma, \alpha\}}
$$

based on which we conclude on the appropriateness of applying the dynamic servicing algorithm.

\section{Conclusion}

The importance of values in this system represents the basis for determining accounting information system during its implementation in military medical institutions. Based on the represented we can conclude that the accounting information system instead of the simple chronological system first came - first served, must be based on dynamic algorithm. This research is focused on development of dynamic, highfrequency, elastic, unique integrated and highly sophisticated accounting information system, which will produce timely, reliable and secure information for military medical institutions. The information system demands the possibility of processing accounting data with the goal of quality improvement of financial reports.

\section{References}

[1] Avi-Itzhak B, Levy H, Raz D. Quantifying fairness in queuing systems. Probability in the Engineering and Informational Sciences 2008; 22(04): 495-517.

[2] Cvetković D, Čangalović $M$, Dugošija D, Kovačević-Vujčić V, Simić S, Vuleta J. Combinatorial optimization. Operations Research Society of Yugoslavia. Belgrade: DOPIS; 1996.

[3] Helbing D, Treiber M, Kesting A. Understanding interarrival and interdeparture time statistics from interactions in queuing systems. Physica A: Statistical Mechanics and its Applications 2006; 363(1): 62-72.

[4] Salehi M, Rostami V, Mogadam A. Usefulness of accounting information system in emerging economy: Empirical evidence of Iran. International Journal of Economics and Finance 2010; 2(2): 186-193.

[5] Milojević I, Mihajlović M, Cvijanović M, Impact of organizational failure of relevance consolidated budget, Agricultural Economics 2012; 59(1): 63-71.

[6] Gnedenko BV. The Theory of Probability and the Elements of Statistics. American Mathematical Soc. 2005; 132(2): 68-74. 
[7] Tsitsiklis JN, Xu K. Queueing system topologies with limited flexibility. Performance Evaluation Review 2013; 41(1): 167-178.

[8] Tsamenyi M, Cullen J, González JMG. Changes in accounting and financial information system in a Spanish electricity company: A new institutional theory analysis. Management Accounting Research 2006; 17(4): 409-432.

[9] Grabski SV, Leech SA, Schmidt PJ. A review of ERP research: A future agenda for accounting information systems. Journal of information systems 2011; 25(1): 37-78.

[10] Brazel JF, Agoglia CP. An Examination of Auditor Planning Judgements in a Complex Accounting Information System Environment*. Contemporary Accounting Research 2007; 24(4): 1059-1083.

[11] Eldenburg L, Soderstrom N, Willis V, Wu A. Behavioral changes following the collaborative development of an accounting information system. Accounting, Organizations and Society 2010; 35(2): 222-237.

[12] Le Boudec JY, Thiran P. Network calculus: a theory of deterministic queuing systems for the internet. Springer Science \& Business Media 2001; 20(5): 123-135.

[13] Gnedenko BV, Kolmogorov AN. Independent Random Variables. Cambridge (Massachusetts): Addison-Wesley; 1954.

[14] Bassamboo A, Randhawa RS, Mieghem JAV. A little flexibility is all you need: on the asymptotic value of flexible capacity in parallel queuing systems. Operations Research 2012; 60(6): 1423-1435.

[15] Hollander A, Denna E, Cherrington JO. Accounting, information technology, and business solutions. Columbus (OH): McGraw-Hill Higher Education; 1999.

[16] Heffers H. Analysis of First-Come First-Served Queuing Systems With Peaked Inputs. Bell System Technical Journal 1973; 52(7): 1215-1228.

[17] Milojević I. Accounting. Belgrade: Center for Economic and Financial Research, 2010.

[18] Meo FD. Overinvestment, subsequent earnings management, and CEO tenure. Spanish Journal of Finance and Accounting 2014; 43(3): 217-240.

[19] Simijonović S. Internal control and internal control components. ODITOR 2016; 2(1): 29-33. 